\title{
The top $3^{+}$gene is essential in Schizosaccharomyces pombe and the lethality associated with its loss is caused by Rad12 helicase activity
}

\author{
Mohamed Maftahi', Christine S. Han', Lance D. Langston', Justin C. Hope ${ }^{2}$, Nico Zigouras ${ }^{1}$ \\ and Greg A. Freyer ${ }^{1,2, *}$ \\ 1Division of Environmental Sciences, Joseph Mailman School of Public Health and 2Department of Anatomy and Cell \\ Biology, College of Physicians and Surgeons, Columbia University, New York, NY 10032, USA
}

Received September 1, 1999; Revised and Accepted October 26, 1999

DDBJ/EMBL/GenBank accession no. AF126287

\begin{abstract}
The topoisomerase III gene (top3+) from Schizosaccharomyces pombe was isolated and a targeted gene disruption (top3::kan ${ }^{\mathrm{R}}$ ) was used to make a diploid strain heterozygous for top $3^{+}$. The diploid was sporulated and the top3:: $k^{\mathrm{R}} \mathrm{n}^{\mathrm{R}}$ spores went through four to eight cell divisions before arresting as elongated, predominantly binucleated cells with incompletely segregated chromosomes. This demonstrates that top $3^{+}$is essential for vegetative growth in fission yeast. The aberrant chromosomal segregation seen in top3:: $k a^{R}$ cells is unlike the 'cut' phenotype seen in mitosis-defective mutants and so we refer to this phenotype as 'torn'. A deletion mutant, rad12-hd (rad12 is a homolog of Saccharomyces cerevisiae SGS1), partially suppressed the lethality of top3 mutants. A point mutant, rad12-K547I, which presumably eliminates helicase activity, also suppresses the lethality of top3 mutants, demonstrating that the lethality seen in top3 cells is most likely caused by the helicase activity of Rad12. This double mutant grows very slowly and has much lower viability compared to rad12-hd top3::kan ${ }^{\mathrm{R}}$ cells, implying that the helicase activity of Rad12 is not the only cause of top3- lethality. The low viability of rad12- top3mutants compared with rad12 single mutants suggests that Top3 also functions independently of Rad12.
\end{abstract}

\section{INTRODUCTION}

The exact role of topoisomerase III (Topo III) in cells is not completely understood. Originally discovered in bacteria, it has now been shown to exist in other organisms, including humans and mice $(1,2)$. In Escherichia coli, Topo III has been shown to possess potent decatenating activity, particularly when single-stranded (ss)DNA is present in the molecules (3).
Escherichia coli Topo III removes negative supercoils, an activity that also requires ssDNA in the substrate (3). More recently it was shown that E.coli RecQ helicase activity stimulates Topo III to fully catenate plasmid DNA (4). RecQ helicase was shown to bind closed circular DNA, presumably creating a single-stranded region for Topo III to catenate double-stranded (ds)DNA molecules. The presence of a singlestranded region in the DNA substrate was not sufficient to stimulate catenation by Topo III, supporting a specific requirement for RecQ helicase in this reaction (4).

Eukaryotic Topo III is a type I topoisomerase and a member of the E.coli type I topoisomerase family, which includes E.coli topoisomerases I and III (1,5-7). The first description of Topo III in eukaryotic cells came from a screen of hyperrecombination mutants in Saccharomyces cerevisiae (7). A mutant was isolated that showed hyper-recombination as well as slow growth. In addition, this mutant was found to be sterile. Isolation of the gene revealed that it shared homology with the E.coli topA and topB genes (the genes coding for topoisomerases I and III, respectively). This homology was confirmed by the ability of the bacterial topA gene to complement the slow growth phenotype of the yeast mutant (7). These data, along with the finding that the gene shared no homology with other eukaryotic topoisomerase genes, led the authors to name it TOP3. In vitro, the TOP3 gene product was shown to weakly relax negatively but not positively supercoiled DNA (5). These investigators also observed that Topo III has a strong preference for binding to ssDNA and that it is a relatively weak topoisomerase. Their studies also considered the activities of E.coli topoisomerases I and III and concluded that biochemically it is more similar to bacterial Topo III. In mice, Topo III is widely expressed in several tissues $(2,8)$. Extremely high levels of Topo III mRNA were detected in mouse testis beginning some 12-14 days after birth. Knockout mice die as embryos, suggesting that Topo III is an essential gene in early mouse development (6).

A screen for extragenic suppressors of the top3 mutant phenotype in S.cerevisiae led to isolation of a gene designated $S G S 1$, for slow growth suppressor (9). Two-hybrid studies showed that the gene product of SGS1 interacts with both 
Table 1. Schizosaccharomyces pombe strains used in this study

\begin{tabular}{|c|c|}
\hline Strain (laboratory) name & Genotype \\
\hline 972 (SZ06) & $h^{-\mathrm{S}}$ \\
\hline $\operatorname{rad12::hd(\mathrm {SZ36})}$ & $h^{-\mathrm{S}}$, ade6-210, leu1-32, ura4-D18, rad12::ura $4^{+}$ \\
\hline top $3^{+} /$top $3:: k a n^{\mathrm{R}}(\mathrm{SZ} 107)$ & $h^{+\mathrm{N}} h^{-\mathrm{S}}$ ura4-D18/ura4-D18, leu1-32/leu1-32, adeM210/adeM216, top3::kmx ${ }^{+} /$top $3^{+}$ \\
\hline rad12-hd top3::kan ${ }^{\mathrm{R}}(\mathrm{SZ109})$ & $h^{-\mathrm{S}}$, ade6-210, leu1-32, ura4-D18, rad12::ura4 $4^{+}$, top $3:: k m x^{+}$ \\
\hline FC584 (SZ106) & $h^{+\mathrm{N}} h^{-\mathrm{S}}$ ura4/ura4, leu1-32/leu1-32, adeM210/adeM216 \\
\hline rad12-K547I (SZ123) & $h^{-\mathrm{S}}$, ade6-210, leu1-32, ura4-D18, rad12-K547I \\
\hline rad12-K547I top3::kan ${ }^{\mathrm{R}}(\mathrm{SZ124})$ & $h^{-\mathrm{S}}$, ade6-210, leu1-32, ura4-D18, rad12-K547I, top3::kmx ${ }^{+}$ \\
\hline
\end{tabular}

All the strains are isogenic to 972 , except the SZ109 diploid strain, which was used in the top $3^{+}$disruption experiment.

topoisomerases II and III $(9,10)$. Sgs1 was shown to be a helicase of the RecQ family $(9,10)$. In E.coli, loss of RecQ helicase has been associated with increased illegitimate recombination (11). In association with other mutations, RecQ mutants are UV sensitive and have greatly reduced homologous recombination $(12,13)$. Sgs1 is three times the size of RecQ, possessing additional sequences both $\mathrm{N}$ - and $\mathrm{C}$-terminal to the centrally located helicase. This family has since been expanded to include the human $B L M$ and $W R N$ genes (associated with Bloom's and Werner's syndromes, respectively), human $R E C Q$ ( $h R E C Q$ ) and two other recently identified human genes containing recQ helicase domains (14-19). The physical association between Topo III and Sgs1 suggests a functional relationship between these two proteins, where Sgs1 creates products whose resolution depends on Topo III. This suggestion is based on the fact that the slow growth seen in Top3deficient cells is suppressed by loss of the Sgs1 helicase.

In the fission yeast Schizosaccharomyces pombe, the rad12+ gene (also referred to as $r q h 1^{+}$and $h u s 2^{+}$) has been isolated and shown to be another member of the RecQ helicase family $(20,21)$. This gene was initially identified in a screen for radiation-sensitive mutants (22) and was independently isolated as $h u s 2^{+}$in a screen for hydroxyurea (HU)-sensitive mutations (23). Our interest in $\mathrm{radl2}^{+}$began with the finding that the rad12-502 mutant has reduced levels of Uve1 activity, a UV damage endonuclease (24). rad12- cells are UV- and $\gamma$-radiation-sensitive as well as sensitive to $\mathrm{HU}(20,21,23)$. In addition, rad12 mutants show chromosomal instability and demonstrate a defect in exiting a HU-induced checkpoint $(20,21)$. This defect was manifested in cells as missegregated chromosomes where DNA is seen stretching between paired nuclei in elongated cells. Because of the relationship of Sgs1 and Top3, we sought to understand whether a similar association existed between Rad12 and Top3 in S.pombe. Here we describe the isolation of S.pombe top $3^{+}$and show that it is essential. Interestingly, the phenotype that we observe in top $3:: \mathrm{kan}^{\mathrm{R}}$ cells is reminiscent of the chromosomal missegregation seen in rad12- cells upon exit from $\mathrm{S}$ phase checkpoint arrest. To distinguish this phenotype from 'cut', where the septum splits the nucleus, we suggest the term 'torn' to describe this phenotype, referring to the ripped appearance of the chromosomes and the absence of an obvious septum in these cells. We further show that the lethality of top $3:: \mathrm{kan}^{\mathrm{R}}$ cells is suppressed in rad12 mutants and present evidence that the lethality is partially dependent on Rad12 helicase activity, although helicase activity alone may not fully explain the lethality. Finally, rad12 mutants do not completely restore viability in top3 mutants, demonstrating that Top3 may also function independently of Rad12.

\section{MATERIALS AND METHODS}

\section{Media and strains}

Media. The sporulation medium was EMM with no nitrogen, supplemented as appropriate. YEA was $30 \mathrm{~g} / \mathrm{l}$ glucose, $5 \mathrm{~g} / \mathrm{l}$ yeast extract and $75 \mathrm{mg} / \mathrm{l}$ adenine. Minimal medium was $1.7 \mathrm{~g}$ of yeast nitrogen base (Bufferad) and $5 \mathrm{~g}\left(\mathrm{NH}_{4}\right)_{2} \mathrm{SO}_{4}$, supplemented with $75 \mu \mathrm{g} / \mathrm{ml}$ uracil, $75 \mu \mathrm{g} / \mathrm{ml}$ leucine and $75 \mu \mathrm{g} / \mathrm{ml}$ adenine unless indicated otherwise.

Strains. All strains used in this study are shown in Table 1.

\section{Plasmids and strain constructions}

Cloning of S.pombe top $3^{+}$cDNA. A partial cDNA for top $3^{+}$ was isolated by PCR amplification of a $292 \mathrm{bp}$ fragment using degenerate primers (Top3sp5' and Top3sp3') based on two highly conserved sequences in the S.cerevisiae and human TOP3 genes. (All oligonucleotides used in this study are shown in Table 2.) The template was $1 \mu \mathrm{g}$ of plasmid DNA isolated from a S.pombe cDNA library. The PCR product was sequenced and the results used to search the S.pombe genome database at the Sanger Center. This search identified a cosmid (c16g5) containing a probable top 3 gene. Likely exons and intron boundaries were identified by comparing the open reading frames from this cosmid with the sequence of S.cerevisiae TOP3.

To isolate a full-length cDNA, $1 \mu \mathrm{g}$ of a S.pombe cDNA library was used as template for PCR amplification using $P f u$ polymerase (Stratagene) and primers (cTOPA and cTOPD) predicted to bracket the complete coding region of top $3^{+}$. The products were gel purified, digested with $N c o I$ and SalI, and cloned into pBluescript (Stratagene). The resulting cDNA, designated pMM1, was completely sequenced using oligonucleotide primers, whose sequences were based on the genomic sequence.

top $3^{+}$genomic clone. A library made by partial HindIII digestion of S.pombe genomic DNA was screened with a radiolabeled probe for clones containing top $3^{+}$sequences. A plasmid (pgTOP3) identified in this screen was shown to contain the entire top $3^{+}$gene by restriction analysis and by PCR amplification of the $5^{\prime}$-end, middle and $3^{\prime}$-end of the gene 
Table 2. Oligonucleotides used in this study

\begin{tabular}{|c|c|c|}
\hline Oligonucleotide & Sequence & Purpose \\
\hline Top3sp5 $5^{\prime a}$ & TGCCAGTTYCCHACNCTBGGCTTTG $^{\mathrm{b}}$ & Degenerate oligo used to isolate top $3^{+}$ \\
\hline Top3sp3'a & CTGTTCKWGGATAAGAWATRAAMCC ${ }^{c}$ & Degenerate oligo used to isolate top $3^{+}$ \\
\hline cTOPA $^{d}$ & AAGGCAGTCCATGGCTATGCGCGTCCTATGTGTTGCTG & Primes at $5^{\prime}$ ATG of top $3+$ to make full-length cDNA \\
\hline cTOPD $^{d}$ & CCGCGAAGTCGACCTAGGTTTGCGGTTCATTATGAC & Primes at $3^{\prime}$ stop of top $3^{+}$to make full-length cDNA \\
\hline $\mathrm{cTOP}^{\mathrm{d}}$ & ATGCGCGTCCTATGTGTTGC & Verify genomic clone \\
\hline $\mathrm{cTOP}^{\mathrm{d}}$ & GTGATGATTTGAGCATCG & Verify genomic clone \\
\hline cTOP $11^{\mathrm{d}}$ & CGGGACAGATGCTACTATG & Verify genomic clone \\
\hline TOP3ver2 & ACGGATCCAACTCAAGATG & Verify genomic clone and TOP3 disruption \\
\hline сTOP9d & CCAAAGGAGCCGAAACG & Verify genomic clone \\
\hline cTOP13 & GGATCATATGAACTAGAAC & Verify genomic clone \\
\hline top3cNotI & ACGAGGCAGCGGCCGCGACGCTTAAAAACGAAAG & Create top $3^{+}$disruption \\
\hline top3dSpeI & CGATGCACACTAGTGGCTTCCGTAATAA & Create top $3^{+}$disruption \\
\hline top3e & GGTTAATCGCACCTCC & Create top $3^{+}$disruption \\
\hline top3f & CTTGGATATTGCACACC & Create top $3^{+}$disruption \\
\hline TOP3ver1 & CGGCTGCAAGGTCTTCGTC & Verify top $3^{+}$disruption \\
\hline Kan1 & GCGGCGTGGGGACAATTC & Verify top $3^{+}$disruption \\
\hline KanRev & CCTCGACATCATCTGCCC & Verify top $3^{+}$disruption \\
\hline
\end{tabular}

\footnotetext{
${ }^{a}$ The degenerate oligonucleotides were designed using the codon bias of S.pombe.

by $=\mathrm{c}+\mathrm{t} ; \mathrm{h}=\mathrm{a}+\mathrm{c}+\mathrm{t} ; \mathrm{n}=\mathrm{a}+\mathrm{c}+\mathrm{t}+\mathrm{g} ; \mathrm{b}=\mathrm{c}+\mathrm{t}+\mathrm{g}$.

${ }^{c} \mathrm{~W}=\mathrm{a}+\mathrm{t} ; \mathrm{r}=\mathrm{a}+\mathrm{g} ; \mathrm{m}=\mathrm{a}+\mathrm{c}$.

${ }^{\mathrm{d}}$ These oligonucleotides were designed using the top $3 \mathrm{cDNA}$ sequence.
}

using paired primers cTOP1/cTOP3, cTOP11/TOP3ver2 and cTOP9/cTOP13, respectively. pgTOP3 was further digested with $\mathrm{BamHI}$ to eliminate sequences downstream of the gene, creating pgTOP3b. Restriction analysis showed that pgTOP3b contains $316 \mathrm{bp}$ of genomic sequence upstream of the ATG start codon and 123 bp downstream from the TAG stop codon.

\section{Construction of the top3 disrupted strains}

The top $3^{+}$gene was disrupted with the kanamycin resistance gene under the control of the TEF promoter (25). The 3'sequences of the top $3^{+}$gene, from nucleotide 1298 to 2261 , were PCR amplified using primers top3cNotI and top3dSpeI. This $963 \mathrm{bp}$ fragment was subcloned into the SmaI site of pBluescript, amplified, cut from there with EcoRI/SpeI (as a $618 \mathrm{bp}$ fragment as there is an internal EcoRI site at nucleotide 1643) and cloned into the EcoRI and SpeI sites downstream of the $k a n^{\mathrm{r}}$ gene in plasmid pFA-kanMX4, creating pkanMX4top3.3' (25). This plasmid contains the E.coli transposon Tn903 $k^{2}{ }^{r}$ fused to the transcriptional and translational control sequences of the TEF gene of the fungus Ashbya gossypii. The $5^{\prime}$-upstream region of top $3^{+}$, from -951 to -25 , was PCR amplified using primers top3e and top3f. This $933 \mathrm{bp}$ fragment was cloned into the SmaI site of pBluescript, amplified, then excised from there with BamHI/Sall and cloned into the BglII and SalI sites upstream of the $\mathrm{kan}^{\mathrm{R}}$ gene in pkanMX4top3.3'. The final construct was designated pTop3Kmx.

The disruption cassette was cut from pTop3Kmx with NotI and was used to transform the diploid strain FC584 (F. Chang) as described (26). Positive colonies were selected for on rich medium containing Geneticin (G418; Gibco). Several colonies were isolated. Proper disruption of top $3^{+}$was confirmed by PCR. The $5^{\prime}$-junction was confirmed using primers TOP3ver1 and Kan1 and the 3 '-junction was confirmed using primers
TOP3ver2 and KanRev. The diploid strain was allowed to sporulate by streaking onto sporulation medium. Tetrads were dissected on YEA plates.

The rad12-hd top $3:: \mathrm{kan}^{\mathrm{R}}$ and rad12-K547I top $3:: \mathrm{kan}^{\mathrm{R}}$ double mutants were made by transforming rad12-hd and rad12-K547I, respectively, with NotI-digested pTop3Kmx. Transformants were selected for on rich medium in the presence of G418. Confirmation of the disruption was carried out by PCR analysis.

\section{Growth kinetics}

Overnight cultures grown in rich medium were diluted $\sim 1: 1000$ and allowed to grow at $30^{\circ} \mathrm{C}$ for $\sim 14 \mathrm{~h}$. The experiment was started when there were $\sim 2 \times 10^{5}$ cells $/ \mathrm{ml}$. Beginning at time 0 and then at each hour for the next $8 \mathrm{~h}, 100 \mu \mathrm{l}$ of cells was placed in $1 \mathrm{ml}$ of isotonic solution and counted in a Coulter counter. The particle size cut-off was set at $6 \mu \mathrm{m}$. Each time point was counted three times. Fold increase was calculated as relative to the first time point, time 0 . Plots are averages of three independent experiments.

\section{Photographs of mutant cells}

Diploid top $3:: \mathrm{kan}^{\mathrm{R}}$ were plated on sporulation plates and incubated for $48 \mathrm{~h}$ at $30^{\circ} \mathrm{C}$. Asci were scraped from the plates and resuspended in $1 \mathrm{ml}$ sterile water with $5 \mu \mathrm{l}$ glusulase. Following incubation for $8 \mathrm{~h}$ at $30^{\circ} \mathrm{C}$, spores were plated onto rich medium plates and incubated for 36 or $72 \mathrm{~h}$ at $30^{\circ} \mathrm{C}$. At $36 \mathrm{~h}$ a mixture of top $3^{+}$and top $3:: \mathrm{kan}^{\mathrm{R}}$ colonies were scraped from the plates and resuspended in water, fixed in ethanol and stained with DAPI at $0.25 \mu \mathrm{g} / \mu \mathrm{l}$. At $72 \mathrm{~h}$ microcolonies, assumed to be top $3:: k a n^{\mathrm{R}}$, were plucked from the plates, fixed and stained with DAPI. All of the radl2 single mutant and rad12 top3 double mutant strains were also examined by 
fluorescence microscopy following DAPI staining. Cells were prepared from cultures grown to mid-log.

\section{RESULTS}

\section{Identifying S.pombe top $3^{+}$}

To isolate the gene for top $3^{+}$in S.pombe, we first used degenerate oligonucleotides as primers (Top3sp5' and Top3sp3') to PCR amplify a fragment of top $3^{+}$using a cDNA library as the template. The oligonucleotide sequences were based on two highly conserved amino acid sequences in the human and S.cerevisiae TOP3 genes. We then sequenced the products of these PCR reactions and used the sequences to search the S.pombe genome database at the Sanger Centre. This search identified a cosmid, c16G5 (GenBank accession no. AL023554) from chromosome II, which contained a probable S.pombe top $3^{+}$homolog. Using the genomic sequence data, we developed primers to amplify a full-length top $3^{+} \mathrm{cDNA}$ using plasmid DNA generated from a cDNA library as template. The cDNA was fully sequenced and the data compared with the genomic sequence to check for fidelity. The coding sequence (GenBank accession no. AAD22485) was used to identify the five introns in top $3^{+}$(Fig. 1).

The complete genomic sequence of top $3^{+}$from ATG to the stop codon is $2203 \mathrm{bp}$ and the complete coding sequence (including the stop) is $1869 \mathrm{bp}$, which translates to a predicted protein of 622 amino acids of $\sim 71.2 \mathrm{kDa}$. BLAST searches of the GenBank database reveal that S.pombe Top3 shares significant sequence homology with topoisomerases in organisms ranging from bacteria to humans (Table 3). An alignment of S.pombe Top3 with human and S.cerevisiae is also shown in Figure 1. Note that the human protein is larger, with 30 additional amino acids at the $\mathrm{N}$-terminus and extending 350 amino acids longer at the $\mathrm{C}$-terminus.

\section{Dissecting top $3^{+}$function}

To investigate the role of Top3 in S.pombe cells, we created a top3 null mutant. We began by disrupting top $3^{+}$with a selectable marker, $k a n^{\mathrm{R}}$, the gene for kanamycin resistance, under control of the TEF promoter (25). The disruption construct, designed to put $\mathrm{kan}^{\mathrm{R}}$ in place of nucleotides -25 to 1643 of genomic top $3^{+}$(Fig. 2A), was used to transform S.pombe diploid strain FC584. Transformants (designated top $3^{+} /$top $3:: \mathrm{kan}^{\mathrm{R}}$ ) were selected for on rich medium containing G418 and specific integration of the $\mathrm{kan}^{\mathrm{R}}$ marker at the top $3^{+}$ locus was confirmed by PCR.

The heterozygous diploid was allowed to sporulate and tetrad analysis showed 2:0 segregation (Fig. 2B). In this picture, four out of 12 tetrads give only one colony. This result was seen in several experiments, showing reduced spore viability in the top $3^{+} / t_{o p} 3:: \mathrm{kan}^{\mathrm{R}}$ diploid heterozygote. Microscopic examination of the spores which failed to form colonies showed that the cells stopped dividing after approximately four to eight cell divisions, indicating that top $3^{+}$is essential for vegetative growth in S.pombe. To better understand the cause of cell death in top $3^{-}$cells, DAPI stained cells were examined by fluorescence microscopy. The top $3^{+} /$top $3:: \mathrm{kan}^{\mathrm{R}}$ diploid strain was sporulated and treated with glusulase to release individual spores. To see early events, cells were scraped from plates that had been incubated for $36 \mathrm{~h}$. This mixture of top $3^{+}$

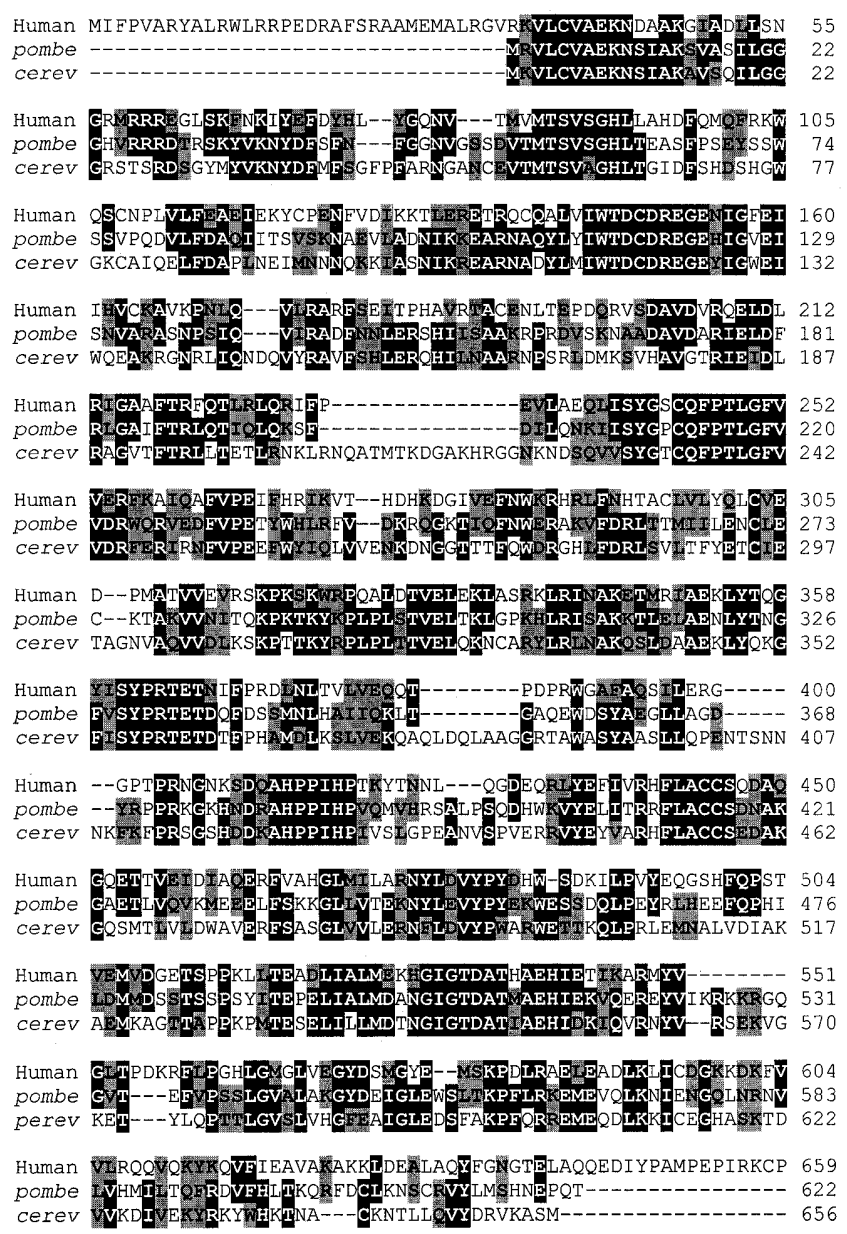

Figure 1. Comparison of the fission yeast, human and S.cerevisiae Top3 amino acid sequences. Schizosaccharomyces pombe Top3 is 622 amino acids long, comparable to the length of S.cerevisiae topo III, which contains 656 amino acids. While both share significant homology with human topo III, the human protein is 1001 amino acids long. Black boxes show areas of identity and gray boxes are areas of similarity.

and top $3:: k a n^{\mathrm{R}}$ cells was examined by fluorescence microscopy. Many cells appeared to be unable to properly segregate their chromosomes (Fig. 3B-D). These cells were elongated and often binucleated and sometimes had DAPI stained material strung out between the paired nuclei. The appearance of DNA in these cells was reminiscent of what is seen in rad12- cells upon exit from a cell cycle checkpoint-induced arrest $(20,21)$. The chromosomal aberrations seen in top $3^{-}$cells differ from those described in 'cut' mutants where abnormal chromosomal segregation arises when the septum cuts through the nucleus. In Figure 3 there are no visible septa bisecting the cells, rather, the DNA appears to be torn apart and so we propose to call this phenotype 'torn'. Cells grown for $72 \mathrm{~h}$ had progressed to forming visible colonies. Microcolonies, presumed to be top $3:: \mathrm{kan}^{\mathrm{R}}$, were plucked from plates and stained with DAPI. These cells had more pronounced morphological changes, with more severely fragmented chromosomes, acentric nuclei and often had DAPI stained material scattered throughout the cell (Fig. 3E-H). We cannot dismiss the possibility that some of the morphological abnormalities seen in 
Table 3. Comparison of topoisomerase 3 in various organisms

\begin{tabular}{lllll}
\hline Organism/gene & Identity $^{\mathrm{a}}$ & Similarity $^{\mathrm{a}}$ & Region of similarity (no. of amino acids) $^{\text {Genbank accession no. }}$ \\
\hline Saccharomyces cerevisiae TOP3 & $44 \%$ & $60 \%$ & 654 & P13099 \\
Human TOP3 & $42 \%$ & $63 \%$ & 600 & Q13472 \\
Mouse TOP3 & $42 \%$ & $63 \%$ & 598 & BAA25662 \\
Caenorhabditis elegans TOP3 & $40 \%$ & $60 \%$ & 623 & AAC13567 \\
Drosophila melanogaster TOP3 & $34 \%$ & $53 \%$ & 591 & AAD13219 \\
Arabidopsis thaliana TOP3 & $30 \%$ & $47 \%$ & 610 & AAD15404 \\
Bacillus subtilis TOP1 & $23 \%$ & $41 \%$ & 532 & P39814 \\
Escherichia coli TOP1 & $24 \%$ & $42 \%$ & 444 & P06612 \\
\hline
\end{tabular}

aThe percentages of identity and similarity between S.pombe Top3 and its homolog in the corresponding organism.

these cells are simply late events occurring in dying cells. Nonetheless, these results demonstrate that cells lacking Top3 undergo aberrant chromosomal events.

\section{Suppression of top $3^{-}$lethality}

As previously reported, the slow growth of top 3 mutants in S.cerevisiae is suppressed by mutations in the SGS1 gene, the homolog of S.pombe rad $12^{+}$. To investigate whether a similar relationship exists in S.pombe, we deleted the top $3^{+}$gene $\left(\right.$ top $\left.3:: \mathrm{kan}^{\mathrm{R}}\right)$ in a rad12 mutant strain, in which the entire helicase domain and C-terminus of Rad12 were deleted (named rad12-hd for helicase deleted). rad12-hd has been previously referred to as rad12:: $\mathrm{ura}^{+}$and was made by replacing nucleotides 1360-3462 of $\mathrm{radl}^{+}$with $\mathrm{ura4}^{+}$(21; Fig. 4A). The double mutant rad12-hd top $3:: \mathrm{kan}^{\mathrm{R}}$ was viable but did not grow as well as the rad12-hd single mutant (Fig. 5).

To ask specifically if it was the helicase activity of $\operatorname{Rad} 12$ that was responsible for the lethality of top 3 mutants, a point mutation in the ATP binding domain of $\mathrm{rad} 12$ was made. The mutation, rad12-K547I, is within the helicase domain and results in an isoleucine substitution at the invariant lysine at amino acid 547 (Fig. 4A). This lysine is conserved in all of the RecQ helicases that have been sequenced and is part of the Walker A-type nucleotide binding box. This region of the helicase domain has been shown to be important for Rad12 activity as the original rad12-502 mutation is a $\mathrm{T} \rightarrow \mathrm{I}$ change at position 543 (27). The rad12-502 mutant suppresses top $3^{-}$lethality but our previous data suggested that it may retain low activity and so we chose to make the rad12-K547I mutation. In previous studies, this lysine was shown to be essential in the hydrolysis of ATP and substitution of this lysine abolished helicase and ATPase activity in a number of helicases, including RecQ (2832). Substitution of arginine for this lysine allowed ATP binding but blocked hydrolysis of ATP and therefore helicase activity in S.cerevisiae RAD3 protein (32), demonstrating the need for a positively charged residue at this position for ATP binding. Changing this lysine to isoleucine should block ATP hydrolysis and helicase activity. Consistent with this, the rad12-K547I mutant grew slowly (Fig. 5), supporting the notion of deficient helicase activity. The rad12-K547I top $3:: \mathrm{kan}^{\mathrm{R}}$ double mutant was viable, demonstrating that the helicase activity of Rad12 causes lethality in top 3 mutants of S.pombe. However, this mutant had lower growth rates and reduced plating efficiency compared with the rad12-hd top $3:: k a n^{\mathrm{R}}$ double mutant (Fig. 5 and Table 4).
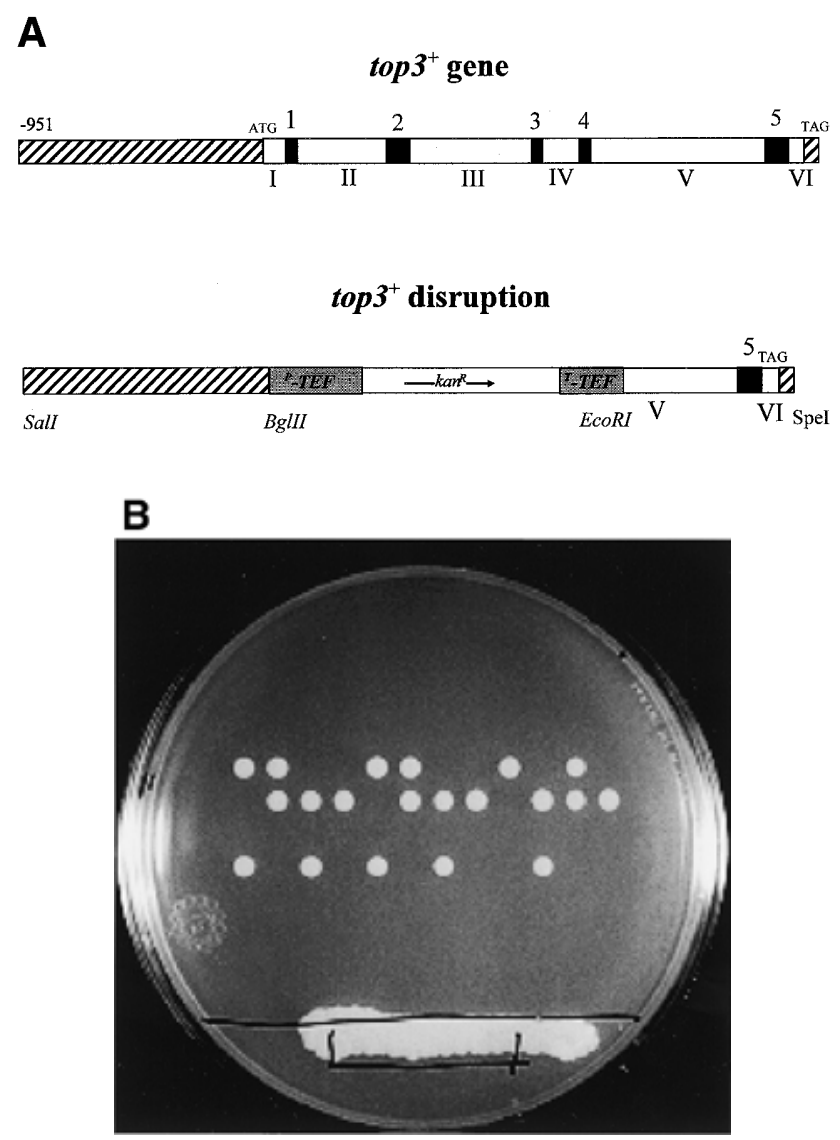

Figure 2. top $^{+}$is an essential gene. (A) PCR-generated fragments from the $5^{\prime}$ and $3^{\prime}$-ends of $t o p 3^{+}$were ligated onto either side of the $k a n^{\mathrm{r}}$ gene within the pFA-kanMX4 plasmid creating pTop3Kmx. The disruption cassette (top3::kan ${ }^{\mathrm{R}}$ ) was cut out of the plasmid with NotI and was used to disrupt the genomic top $3^{+}$gene in a S.pombe diploid strain. Black boxes represent introns (1-5), white boxes are exons (I-VI) and striped boxes show intergenic regions. The kanamycin gene $\left(k a n^{\mathrm{r}}\right)$ is flanked by control sequences of the TEF gene (shown in gray) of the fungus A.gossypii. (B) The diploid strain was sporulated and the asci dissected. Tetrad analysis revealed a 2:0 segregation where only two spores were viable in each tetrad. Some tetrads are shown here with only one viable spore.

We then quantitated the differences in growth rates by studying growth kinetics for each single and double mutant and comparing them with the growth of wild-type S.pombe (972). Growth studies were carried out in three separate 


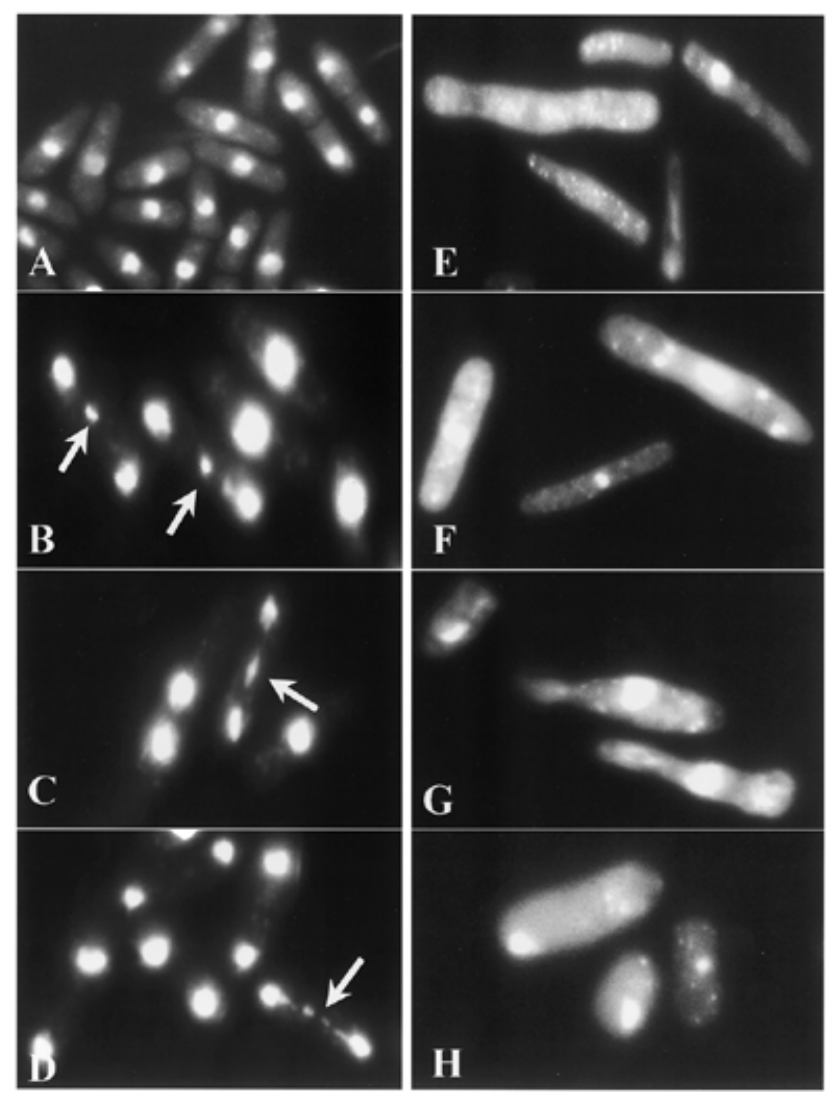

Figure 3. Fluorescence microscopic images of DAPI stained top $3:: k a n^{\mathrm{R}}$ cells. Wild-type cells are shown in (A). A top $3^{+} /$top $3:: \mathrm{kan}^{\mathrm{R}}$ strain was sporulated and treated with glusulase. Then spores were plated and allowed to grow on YEA plates. After $36 \mathrm{~h}$ colonies were too small to isolate individually so cells were scraped from the plates, fixed and stained with DAPI (B-D). These photographs show a mixture of top $3^{+}$and top $3:: \mathrm{kan}^{\mathrm{R}}$ cells. Note the extranuclear fluorescent material and the fragmented nature of chromosomal material in the cells indicated with arrows. After $72 \mathrm{~h}$ microcolonies, assumed to be top $3:: \mathrm{kan}^{\mathrm{R}}$, were isolated, fixed and stained $(\mathbf{E}-\mathbf{H})$. Here the cells have a much more severe phenotype, with very elongated cells and very fragmented fluorescent material. In many cells no nucleus is apparent and instead what appears to be chromosomal material is dispersed throughout the cell.

experiments where all strains were grown simultaneously. The cells were grown at $30^{\circ} \mathrm{C}$ in YEA with shaking. An overnight culture was grown starting with a dilution that would allow cells to reach mid-log by morning. An aliquot of cells was diluted 1:100 and counted in a Coulter counter. We carried out preliminary readings in the range of concentrations needed for these studies using the Coulter counter and showed that it gave identical cell counts to those obtained manually using a hemocytometer. Cell growth was monitored by counting cells every hour for $8 \mathrm{~h}$. The data were very consistent both within and between experiments. As can be seen in Figure 5 and Table 4, both of the rad12 single mutants grew slower than the wildtype. Of these, rad12-hd grew better than the point mutant rad12-K547I, with doubling times of $2 \mathrm{~h} 23 \mathrm{~min}$ and $2 \mathrm{~h}$ $33 \mathrm{~min}$, respectively. When top $3^{+}$was disrupted in rad12-hd, growth slowed only slightly (the doubling time increased from $2 \mathrm{~h} 23 \mathrm{~min}$ to $2 \mathrm{~h} 39 \mathrm{~min}$ ). In contrast, growth in rad12-K547I top3::kan ${ }^{\mathrm{R}}$ was severely reduced, with a doubling time of $3 \mathrm{~h}$ $43 \mathrm{~min}$, which represents a $70 \mathrm{~min}$ increase in doubling time.

\section{Fate of top3- cells}

In addition to cell growth, we also measured cell viability. In three separate experiments cells were grown at $30^{\circ} \mathrm{C}$ in YEA to mid-log and counted. Based on the cell count, an aliquot of each culture was diluted and 200 cells were plated onto YEA plates. The plates were incubated for 3-5 days and counted. A summary of the results are shown in Table 4. The viability is calculated as the fraction of cells that formed colonies (i.e. no. of colonies/no. of cells plated). The average viability for wildtype cells was $90 \%$. Each of the rad12 single mutants showed significantly lower viability; $44 \%$ for rad12-hd and $18 \%$ for rad12-K547I. The viability decreased for each double mutant, dropping to $19 \%$ for rad12-hd top3::kan ${ }^{\mathrm{R}}$ and to only $7 \%$ for rad12-K547I top3::kan ${ }^{\mathrm{R}}$. These viability results parallel the results of the growth studies, in that the viability of each strain was comparable with the growth rate. Thus, of the mutants, rad12-hd has the highest viability with the fastest growth rate and $\mathrm{rad12}-\mathrm{K} 547 \mathrm{I}$ top $3: \mathrm{kan}^{\mathrm{R}}$ has the lowest viability with the slowest growth rate. What these results suggest is that the decrease in growth is not due to cells dividing more slowly, but to more cells dying.

In addition, we fixed and DAPI stained cells from each of the single and double mutants and examined them by fluorescence microscopy. Photographs of these cells are shown in Figure 6. These photographs show that in all mutant strains there is a mix of normal and elongated cells. In addition, some cells have evidence of fragmented chromosomes, revealed by multiple fluorescent spots. Chromosomal fragmentation is typically seen in elongated cells. The strains with the highest proportion of cells having altered morphologies are those with the slowest cell growth and lowest plating efficiency. Thus, comparison of rad12-hd with rad12-K547I top3::kan ${ }^{\mathrm{R}}$ shows that rad12-hd has relatively few abnormal appearing cells while many rad12K547I top3::kan ${ }^{\mathrm{R}}$ cells are elongated, with smaller cells also containing some aberrant chromosomal features such as extranuclear DAPI stained material. Thus, those cells with abnormal morphologies are likely to account for the cells which are dying. There are a greater number of abnormal appearing cells in rad12 top3 double mutants compared with rad12 single mutants, but the abnormalities appear similar.

\section{DISCUSSION}

\section{How do cells mutated in top $3^{+}$die?}

The findings presented here demonstrate that the top $3^{+}$gene is essential for vegetative growth in S.pombe. Immediately after sporulation of a top $3^{+} /$top $3:: \mathrm{kan}^{\mathrm{R}}$ diploid strain, cells lacking Top3 are capable of dividing, but after a few generations cell growth stops. This would appear to be a function of accumulated chromosomal aberrations and not due to depletion of a remaining supply of active Top3 from the top $3^{+}$allele of the diploid. This is based on observing Top3-deficient cells for $30 \mathrm{~h}$ after sporulation. top $3^{+} /$top $3:: \mathrm{kan}^{\mathrm{R}}$ diploid cells were allowed to sporulate and dissected into tetrads. Forty tetrads were followed microscopically for $30 \mathrm{~h}$. What we observed was that elongated cells appeared throughout the growth of colonies, as early as the first cell division. This demonstrates that the effects of loss of Top3 on cells were immediate and not delayed, as would be expected if a supply of Top3 were being 

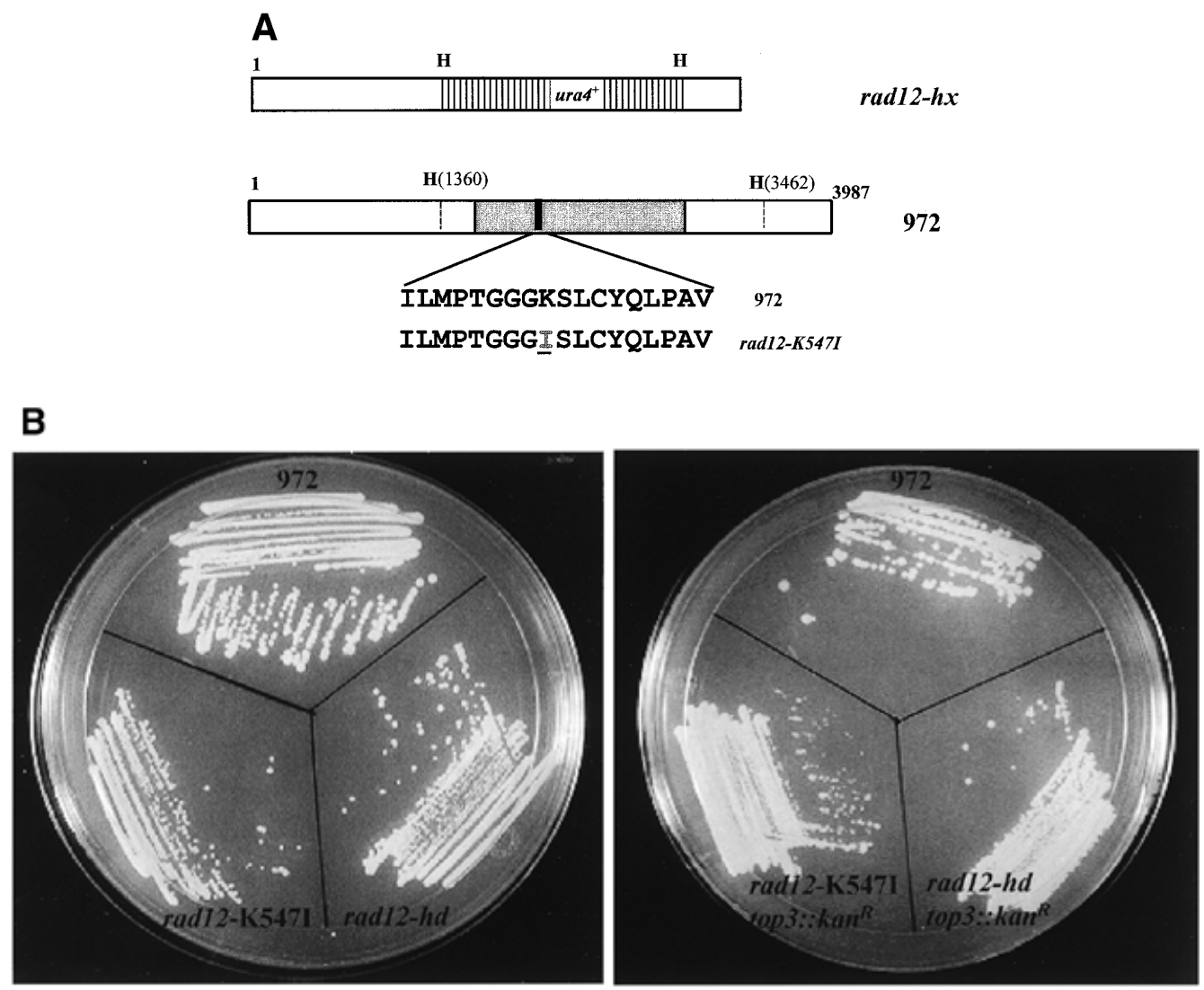

Figure 4. Mutations in rad $12^{+}$suppress the lethality of top 3 mutations. The top $3^{+}$gene was disrupted in two separate rad 12 mutant backgrounds, rad 12 - K547I and rad12-hd, to show that loss of Rad12 helicase activity restores viability in a top3::kan ${ }^{\mathrm{R}}$ mutant. (A) Schematic of the two rad12 mutants. The striped box indicates

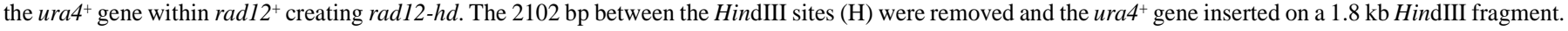
rad12-K547I was created by mutating the invariant lysine (K) at position 547 to an isoleucine (I). The helicase domain is indicated in gray and the Walker A-box in black. (B) Wild-type (972) and mutant strains were streaked onto YEA plates and incubated for $2-3$ days at $30^{\circ} \mathrm{C}$.

Table 4. Growth characteristics of $\mathrm{rad} 12^{-}$and $\mathrm{rad} 12^{-}$top $3^{-}$mutants

\begin{tabular}{|c|c|c|c|c|}
\hline Strain & Average doubling time & Growth rate relative to 972 & Growth rate double/single ${ }^{a}$ & Viability (colonies/cells plated) (range) \\
\hline rad12+ top $^{+}(972)^{\mathrm{c}}$ & $1 \mathrm{~h} 52 \mathrm{~min}$ & - & - & $0.897(0.72-1.16)$ \\
\hline rad12-hd top $3^{+}$ & $2 \mathrm{~h} 23 \mathrm{~min}$ & $78 \%$ & - & $0.312(0.25-0.46)$ \\
\hline rad12-hd top $3:: k m x^{+}$ & 2 h 39 min & $70 \%$ & $90 \%$ & $0.189(0.08-0.25)$ \\
\hline rad12-K547I top $3^{+}$ & $2 \mathrm{~h} 33 \mathrm{~min}$ & $73 \%$ & - & $0.18(0.15-0.22)$ \\
\hline rad12-K547I top3::kmx ${ }^{+}$ & $3 \mathrm{~h} 43 \mathrm{~min}$ & $50 \%$ & $69 \%$ & $0.07(0.05-0.08)$ \\
\hline
\end{tabular}

${ }^{a}$ The growth rate double/single corresponds to the growth of the double mutant rad12- ${ }^{-}$op $3^{-}$relative to its corresponding single mutant rad12-

${ }^{b}$ The viability (colonies/cells plated) corresponds to the plating efficiency.

'In this study 972 is used as the reference strain.

depleted. Also, some colonies accumulated as few as 6-20 cells where others grew to as many as several hundred before growth ceased. If active Top3 was being titrated out we would predict a more uniform number of cells to accumulate in each colony. The assumption that top 3 mutants die as a result of accumulated chromosomal aberrations is based on the observations seen in DAPI stained cells.

It was previously demonstrated that in $\mathrm{rad} 12^{+}$mutants, chromosomal aberrations are readily visualized in cells exiting a cell cycle checkpoint arrest $(20,21)$. These aberrations were observed in DAPI stained dividing cells, where the bulk of the DNA can typically be seen to have separated but some DNA is seen strung out between the two nuclei. This is the same appearance that DNA has in DAPI stained top3::kan ${ }^{\mathrm{R}}$ cells seen in Figure 3B-D. We assume that this extranuclear DNA occurs as a result of unsegregated sister chromatids, similar to what was described in radl $12^{+}$mutants upon exit from an induced $\mathrm{S}$ phase checkpoint arrest, where this was attributed to a defect in the suppression of recombination (20). In these cells there is no evidence that the septum is cleaving the nucleus. 


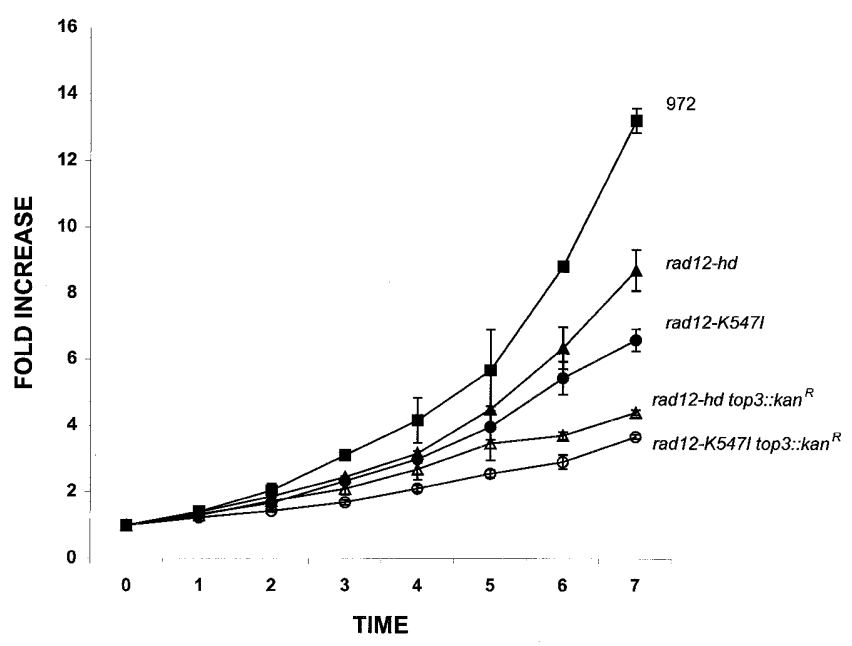

Figure 5. Growth kinetics. YEA was inoculated with each strain at a cell concentration that overnight yielded logarithmic growing cultures of similar cell concentration $\left(0.5 \times 10^{4}-2.0 \times 10^{5}\right)$ the next morning. Aliquots of cell suspensions were taken every hour for $7 \mathrm{~h}$ and the cell count determined at each time point in a Coulter counter. The plot shows the average of three independent experiments. In some cases the error bars are smaller than the symbol and so are not visible. (Square), 972; (filled triangle), rad12- $h d$; (filled circle), rad12K547I; (open triangle), rad12-hd top3::kan ${ }^{\mathrm{R}}$; (open circle), rad12-K547I top $3:: \mathrm{kan}^{\mathrm{R}}$.

We refer to this phenotype as 'torn' to distinguish it from the 'cut' phenotype that occurs in S.pombe cells mutated in top $2^{+}$ or in one of the cut genes (33-36). Microcolonies formed on plates grown for 3 days, presumed to be top $3:: \mathrm{kn}^{\mathrm{R}}$ were picked with the aid of a microscope. Multiple colonies were picked, stained with DAPI and examined microscopically (Fig. $3 \mathrm{E}-\mathrm{H})$. Here we see that the chromosomes appear very degraded with cells having unusual and elongated shapes. DAPI stained material is seen throughout, suggesting fragmented chromosomes. While it is likely that some portion of these alterations are attributable to loss of Top3, these cells are also dead. Therefore, we cannot rule out the possibility that some of the chaotic appearance in these cells is typical of any dying cell. Nonetheless, it would appear that in S.pombe, cells lacking Top3 die as the result of an inability to properly segregate their chromosomes. Finally, it is interesting to note the reduced spore viability in a top $3^{+} /$top $3:: \mathrm{kan}^{\mathrm{R}}$ heterozygous diploid (Fig. 2B). Comparatively, it had also been reported that null mutations of TOP3 in S.cerevisiae display a pleiotropic phenotype including a sporulation defect (9).

\section{Cell viability and growth kinetics}

In this study we have used two parameters to quantitate the ability of rad12 mutants to suppress the lethality of cells lacking Top3. Growth kinetics measure the rate of increase in cell number in a culture in log growth from which we can calculate an average doubling time. Plating efficiency is a measure of cell viability, where we plate a specific number of cells from a mid-log culture, incubate the plates for 3-5 days and count the number of colonies formed. These two measurements are not independent and in fact the slow growth in these mutants may be due to reduced cell viability. As seen in Figure 6 , dividing cells in the mutant strains are similar in size to

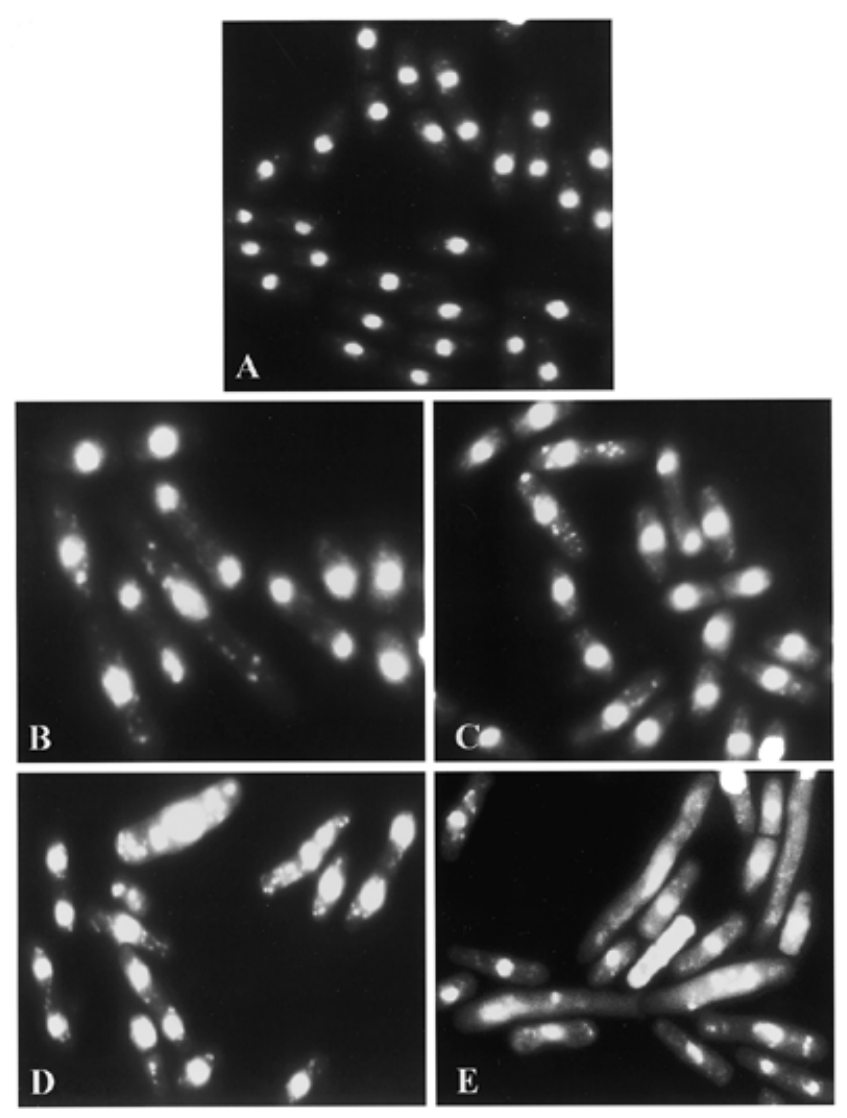

Figure 6. Fluorescence microscopy showing the morphologies of the single and double mutants. Cells were isolated, fixed and stained with DAPI from mid-log cultures of each strain. It is clear that the increased severity and frequency of nuclear abnormalities and cellular morphologies parallels the severity of the phenotype in terms of growth and viability.

dividing wild-type cells. Since cell length is a reflection of cell division time in S.pombe, we conclude that these mutants have the same, or very similar, doubling times as wild-type cells.

\section{Is it the Rad12 helicase activity that causes cell death in top $3^{-}$cells?}

Our results, showing that the lethality seen in top3::kan ${ }^{\mathrm{R}}$ mutants is suppressed in a rad12- background, are reminiscent of the results in S.cerevisiae where the slow growth phenotype of a top3 mutant is suppressed in a $s g s 1$ mutant background (9). These results suggest that the phenotypes associated with loss of Top3 occur because this topoisomerase is needed to resolve some intermediate that is generated by the RecQ helicase activity of Rad12 or Sgs1. This conclusion is in conflict with the results of one report in S.cerevisiae (37). In that study SGS1 was mutated at the invariant lysine in the ATP binding domain within the Walker A-box which is known to completely block ATPase and helicase activity. This mutant, sgsl-hd, was shown to completely lack helicase activity. sgs 1-hd was transformed into a sgs 1 top3 double mutant on a plasmid. The transformed cells were shown to behave as if they were wild-type for $S G S 1$, i.e. the cells grew slowly. These data demonstrate that it is some function other than the helicase that is responsible for the suppression of Top3 activity in S.cerevisiae. 
In our studies, we used two rad12 mutants to suppress top3lethality; rad12-hd has a deletion of the entire helicase domain, while rad12-K547I has a point mutation at the invariant lysine that should eliminate ATP binding and helicase activity (see Fig. 4A). rad12-hd is a null mutant eliminating the terminal two-thirds of the gene. The top $3:: \mathrm{kan}^{\mathrm{R}}$ mutation was introduced into each of these $\mathrm{rad} 12^{-}$strains by gene replacement and the double mutants analyzed for viability and cell growth. Both double mutants were viable, showing that either mutation suppresses the lethal effects of deleting top3. The fact that a point mutation thought to inactivate the helicase activity of Rad12 suppresses loss of Top3 argues that the helicase activity is responsible for this lethality, however, the degree to which these two alleles could suppress the lethality differed greatly. In the case of rad12-hd top $3:: \mathrm{kan}^{\mathrm{R}}$ we found that cell viability was $19 \%$, compared with $31 \%$ for the rad12-hd single mutant. The doubling time for rad12-hd top3::kan ${ }^{\mathrm{R}}$ was $2 \mathrm{~h}$ $39 \mathrm{~min}$, a $17 \mathrm{~min}$ decrease from the rad12-hd single mutant. There is a correspondingly small increase in morphological aberrations seen in the double mutant compared with the single mutant as well. In contrast, rad12-K547I mutants were much less efficient in suppressing the lethality of top3::kan ${ }^{\mathrm{R}}$. rad12-K547I top $3:: \mathrm{kan}^{\mathrm{R}}$ had greatly reduced cell viability, extremely slow cell growth and extreme morphological changes to the cells. The viability of rad12-K547I top3::kan ${ }^{\mathrm{R}}$ was only $8 \%$, with a doubling time of $3 \mathrm{~h}$ $43 \mathrm{~min}$. The reduced viability and growth kinetics are borne out by the extreme level of morphological abnormalities seen in these double mutants (Fig. 6). It should be noted that while it is assumed that the rad12-K547I mutant lacks helicase activity, it is possible that low levels of activity exist and this could be the cause of the reduced viability seen in this mutant compared with the rad12-hd mutant.

From these results we draw three conclusions. First, the helicase is partially responsible for cell lethality in S.pombe top3 mutants, as inactivation of the helicase partially restores viability. Second, some other activity in Rad12 also contributes to the lethality of top 3 mutants, as cell viability and growth are greatly improved when the entire Rad12 is deleted as in rad12-hd top $3:: \mathrm{kan}^{\mathrm{R}}$, compared with simply inactivating the Rad12 helicase as in rad12-K547I top $3::$ kan $^{\mathrm{R}}$. This would partially support the conclusions of Lu et al. (37) in claiming that something other than the Sgs1 helicase causes slow growth in S.cerevisiae top3 mutants. An alternative explanation in S.pombe is that inactive Rad12 binds to its target DNA but with no helicase activity to move it along, it creates a block that is unable to move along the DNA. Third, Top3 appears to have essential functions independent of Rad12 based on the fact that neither rad12 mutant completely suppresses the lethality of top3 mutants. This is seen by comparing growth and viability of each single rad12 mutant with their corresponding double mutant.

\section{Comparison of top3 mutation in S.pombe and S.cerevisiae}

The lethality seen in S.pombe top3 mutants is in contrast to the phenotype of S.cerevisiae top3 mutants which, despite their slow growth compared to the wild-type, are viable. This lethality appears to more closely reflect the situation in $m T O P 3 \alpha$ knockout mice, which die early in embryogenesis even though the mouse genome contains at least one additional top3 homolog, mTOP3 $\beta$ (6). The lethality associated with loss of Top3 in S.pombe is partially suppressed by mutations in rad $12^{+}$, while in S.cerevisiae the slow growth of top3 mutants is completely suppressed by mutations in SGS1. We would not anticipate that rad12 top3 double mutants would grow at wildtype rates as $S$.pombe rad12 single mutants already grow more slowly and have reduced viability compared with wild-type cells. However, the rad12 top 3 double mutants do not recover to the growth rates of the rad12 single mutants. This result demonstrates that Top3 must also function independently of $\operatorname{Rad} 12$. This does not appear to be the case in S.cerevisiae. It may eventually be shown that for Rad12 it has functions that are independent of Top3.

The rad12-hd deletion mutant grows much better than the rad12-K547I point mutant. These data suggest that the presence of Rad12 with an inactivated helicase is detrimental to the cell. Results from both S.pombe and S.cerevisiae support the conclusion that most of the effects associated with loss of Top3 depend on the presence of Rad12/Sgs1. Together, our results demonstrate that Top3 and Rad12 play similar roles to their counterparts in S.cerevisiae, however, differences suggest that they appear to play a more complex role in S.pombe. While there is a large body of data concerning phenotypes associated with loss of the RecQ helicases and Topo III, the actual biological functions of these proteins remain to be determined.

\section{NOTE}

The authors note that during the time in which this work was under review a manuscript similar in scope was published in Nucleic Acids Research (38).

\section{ACKNOWLEDGEMENTS}

We are grateful for the assistance of Ms Eva Lin. We would also like to thank Scott Davey and Justin Weinstein for critical reading of this manuscript. This work was supported by Public Health Service grant CA-72647 from the National Cancer Institute and ES-07940 from the National Institute of Environmental Health Sciences.

\section{REFERENCES}

1. Hanai,R., Caron,P.R. and Wang,J.C. (1996) Proc. Natl Acad. Sci. USA, 93, 3653-3657.

2. Seki,T., Seki,M., Katada,T. and Enomoto,T. (1998) Biochim. Biophys. Acta, 1396, 127-131.

3. DiGate,R.J. and Marians,K.J. (1988) J. Biol. Chem., 263, 13366-13373.

4. Harmon,F.G., DiGate,R.J. and Kowalczykowski,S.C. (1999) Mol. Cell, 3, 611-620.

5. Kim,R.A. and Wang,J.C. (1992) J. Biol. Chem., 267, 17178-17185.

6. Li,W. and Wang,J.C. (1998) Proc. Natl Acad. Sci. USA, 95, 1010-1013.

7. Wallis,J.W., Chrebet,G., Brodsky,G., Rolfe,M. and Rothstein,R. (1989) Cell, 58, 409-419.

8. Seki,T., Wang,W.S., Okumura,N., Seki,M., Katada,T. and Enomoto,T. (1998) Biochim. Biophys. Acta, 1398, 377-381.

9. Gangloff,S., McDonald,J.P., Bendixen,C., Arthur,L. and Rothstein,R. (1994) Mol. Cell. Biol., 14, 8391-8398.

10. Watt,P.M., Louis,E.J., Borts,R.H. and Hickson,I.D. (1995) Cell, 81, 253-260.

11. Hanada,K., Ukita,T., Kohno,Y., Saito,K., Kato,J. and Ikeda,H. (1997) Proc. Natl Acad. Sci. USA, 94, 3860-3865.

12. Nakayama,K., Irino,N. and Nakayama,H. (1985) Mol. Gen. Genet., 200, 266-271.

13. Nakayama,K., Shiota,S. and Nakayama,H. (1988) Can. J. Microbiol., 34, 905-907.

14. Ellis,N.A., Groden,J., Ye,T.Z., Straughen,J., Lennon,D.J., Ciocci,S., Proytcheva,M. and German,J. (1995) Cell, 83, 655-666. 
15. Matsumoto,T., Imamura,O., Yamabe,Y., Kuromitsu,J., Tokutake,Y., Shimamoto,A., Suzuki,N., Satoh,M., Kitao,S., Ichikawa,K., Kataoka,H., Sugawara,K., Thomas,W., Mason,B., Tsuchihashi,Z., Drayna,D., Sugawara,M., Sugimoto,M., Furuichi,Y. and Goto,M. (1997) Hum. Genet., 100, 123-130.

16. Karow,J.K., Chakraverty,R.K. and Hickson,I.D. (1997) J. Biol. Chem., 272, 30611-30614.

17. Gray,M.D., Shen,J.C., Kamath-Loeb,A.S., Blank,A., Sopher,B.L., Martin,G.M., Oshima,J. and Loeb,L.A. (1997) Nature Genet., 17, 100-103.

18. Puranam,K.L. and Blackshear,P.J. (1994) J. Biol. Chem., 269, 29838-29845.

19. Kitao,S., Ohsugi,I., Ichikawa,K., Goto,M., Furuichi,Y. and Shimamoto,A. (1998) Genomics, 54, 443-452.

20. Stewart,E., Chapman,C.R., Al-Khodairy,F., Carr,A.M. and Enoch,T. (1997) EMBO J., 16, 2682-2692.

21. Davey,S., Han,C.S., Ramer,S.A., Klassen,J.C., Jacobson,A., Eisenberger,A., Hopkins,K.M., Lieberman,H.B. and Freyer,G.A. (1998) Mol. Cell. Biol., 18, 2721-2728.

22. Nasim,A. and Smith,B.P. (1975) Genetics, 79, 573-582.

23. Enoch,T., Carr,A.M. and Nurse,P. (1992) Genes Dev., 6, 2035-2046.

24. Freyer,G.A., Davey,S., Ferrer,J.V., Martin,A.M., Beach,D. and Doetsch,P.W. (1995) Mol. Cell. Biol., 15, 4572-4577.

25. Wach,A., Brachat,A., Pohlmann,R. and Philippsen,P. (1994) Yeast, 10, 1793-1808.
26. Bahler,J., Wu,J.Q., Longtine,M.S., Shah,N.G., McKenzie,A., Steever,A.B., Wach,A., Philippsen,P. and Pringle,J.R. (1998) Yeast, 14, 943-951.

27. Murray,J.M., Lindsay,H.D., Munday,C.A. and Carr,A.M. (1997) Mol. Cell. Biol., 17, 6868-6875.

28. Walker,J.E., Saraste,M., Runswick,M.J. and Gay,N.J. (1982) EMBO J., 1, 945-951.

29. Smith,R.H. and Kotin,R.M. (1998) J. Virol., 72, 4874-4881.

30. Ford,M.J., Anton,I.A. and Lane,D.P. (1988) Nature, 332, 736-738.

31. Carles-Kinch,K., George,J.W. and Kreuzer,K.N. (1997) EMBO J., 16, 4142-4151.

32. Sung,P., Higgins,D., Prakash,L. and Prakash,S. (1988) EMBO J., 7, 3263-3269.

33. Uzawa,S., Samejima,I., Hirano,T., Tanaka,K. and Yanagida,M. (1990) Cell, 62, 913-925.

34. Uemura,T. and Yanagida,M. (1984) EMBO J., 3, 1737-1744.

35. Uemura,T. and Yanagida,M. (1986) EMBO J., 5, 1003-1010.

36. Hirano,T., Fukahashi,S., Uemura,T. and Yanagida,M. (1986) EMBO J., 5, 2973-2979.

37. Lu,J., Mullen,J.R., Brill,S.J., Kleff,S., Romeo,A.M. and Sternglanz,R. (1996) Nature, 383, 678-679.

38. Goodwin,A., Wang,S.-W, Toda,T., Norbury,C. and Hickson,I.D. (1999) Nucleic Acids Res., 27, 4050-4058. 